\title{
Oncological outcomes of robotic-assisted versus open pancreatoduodenectomy for pancreatic ductal adenocarcinoma: a propensity score-matched analysis
}

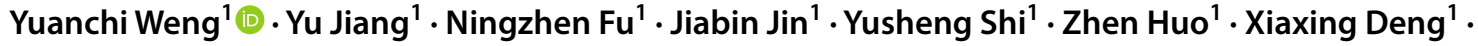 \\ Chenghong Peng ${ }^{1} \cdot$ Baiyong Shen ${ }^{1}$
}

Received: 21 December 2019 / Accepted: 7 July 2020 / Published online: 21 July 2020

(c) The Author(s) 2020

\begin{abstract}
Background Robotic-assisted minimally invasive surgery is associated with worse oncologic outcomes for some but not other types of cancers. We conducted a propensity score-matched analysis to compare oncologic outcomes of robotic-assisted laparoscopic (RPD) vs. open pancreatoduodenectomy (OPD) for pancreatic ductal adenocarcinoma (PDAC).

Methods Treatment-naïve PDAC patients undergoing either RPD or OPD at our hospital between January 2013 and December 2017 were included. Propensity score matching was conducted at a ratio of 1:2. The primary outcome was disease-free survival (DFS) and overall survival (OS).

Results A total of 672 cases were identified. The propensity score-matched cohort included 105 patients receiving RPD and 210 patients receiving OPD. The 2 groups did not differ in the number of retrieved lymph nodes [11 (7-16) vs. 11 (6-17), $P=0.622]$ and $\mathrm{R} 0$ resection rate $(88.6 \%$ vs. $89.0 \%, P=0.899)$. There was no statistically significant difference in median DFS (14 [95\% CI 11-22] vs. 12 [95\% CI 10-14] months (HR 0.94; 95\% CI 0.87-1.50; log-rank $P=0.345$ ) and median OS (27 [95\% CI 22-35] vs. 20 [95\% CI 18-24] months (HR 0.77; 95\% CI 0.57-1.04; log-rank $P=0.087$ ) between the two groups. Multivariate COX analysis showed that RPD was not an independent predictor of DFS (HR 0.90; 95\% CI 0.68-1.19, $P=0.456$ ) or OS (HR 0.77; 95\% CI 0.57-1.05, $P=0.094$ ).

Conclusion Comparable DFS and OS were observed between patients receiving RPD and OPD. This preliminary finding requires further confirmation with prospective randomized controlled trials.
\end{abstract}

Keywords Pancreatic ductal adenocarcinoma $\cdot$ Robotic-assisted pancreatoduodenectomy $\cdot$ Open pancreatoduodenectomy · Propensity score matching

Open pancreatoduodenectomy (OPD) is the cornerstone in the treatment of pancreatic ductal adenocarcinoma (PDAC)

Yuanchi Weng, Yu Jiang, and Ningzhen Fu have contributed equally to this work.

Xiaxing Deng

kejiadxx@hotmail.com

$\triangle$ Chenghong Peng

chhpeng@yeah.net

Baiyong Shen

shenby@shsmu.edu.cn

1 Department of General Surgery, Pancreatic Disease Center, Ruijin Hospital, Shanghai Jiao Tong University School of Medicine, No. 197 Ruijin Er Road, Huangpu District, Shanghai 200025, China in the pancreatic head or uncinate process, but is associated with significant morbidities [1-4]. Minimally invasive surgery was introduced in 1994 for PDAC patients [5], followed by robotic-assisted pancreatoduodenectomy (RPD) in 2003 [6]. Though technically challenging, RPD has a variety of advantages, including less blood loss, faster recovery, and less postoperative complications [4, 7-12]. However, the long-term oncological outcomes of RPD remain undefined [13-17].

Propensity score matching is a statistical method to minimize bias in retrospective studies [18]. We conducted a retrospective analysis to compare the long-term oncological outcomes between RPD and OPD using propensity scorematching. The primary end point of the study was diseasefree survival (DFS) and overall survival (OS). 


\section{Methods}

The study was undertaken according to the Strengthening the Reporting of Observational Studies in Epidemiology (STROBE) guidelines [19] and in accordance with the latest version of the Declaration of Helsinki. The study protocol was approved by the Institutional Review Board, Ruijin Hospital. Informed consent was waived since the study was observational and retrospective.

\section{Patient selection and treatment}

We conducted this retrospective propensity score matched cohort study from a prospective database, and included treatment-naïve PDAC patients who underwent RPD or OPD between January 2013 and December 2017 at the Pancreatic Surgery Department of Ruijin Hospital affiliated to Shanghai Jiaotong University School of Medicine. The RPD and OPD cases included in this study were performed by the same group of surgeons, who had experience of OPD for more than 1000 cases and experience of laparoscopic pancreatic surgery for more than 120 cases. The selection of RPD was based on our surgical team's suggestion, we provided robotic approach choice for the following patients: (1) Stage I or II PDAC cases without "borderline resectable" lesions; (2) Preoperative serum total bilirubin $\leq 250 \mu \mathrm{mol} / \mathrm{L}$; (3) Patients less than 90 years old; (4) ASA score I-III; (5) Without complicated major abdominal surgery history; (6) Without contraindication of pneumoperitoneum. Finally, patients made their choice according to their preference and acceptance of the robotic approach. Our initial cases of RPD (from 2010 to 2012), as cases surpassing the learning curve according to previous study about the learning curve of RPD, were not included [20]. The diagnosis of PDAC was established according to the NCCN Guidelines for Pancreatic Adenocarcinoma on the basis of differential diagnosis to exclude mucous cystadenocarcinoma, signet ring cell carcinomas, adenosquamous carcinomas, undifferentiated (anaplastic) carcinomas, and mixed ductal-endocrine carcinomas [21]. PDAC was staged based on the AJCC 8th TNM stage manual [22]. Major exclusion criteria were: (1) heterogeneous carcinoma (e.g., intraductal papillary mucinous tumor or pancreatic adenosquamous carcinoma), (2) T4 and/or M1 disease, and (3) missing key clinical variables or followup data.

After passing the learning curve of RPD, our surgical team concluded a standard surgical process. The detailed surgical procedures and lymphadenectomy are described in our previous study [23]. After laparoscopic exploration, if diagnosed as T4 or M1 stage, curative surgery would not been performed, and patients would receive subsequent chemotherapy. The "artery first" approach was used when tumors were adjacent to superior mesenteric artery and when portal vein (PV) or superior mesenteric vein (SMV) were involved. $\mathrm{PV} / \mathrm{SMV}$ wedge resection and repair or end-to-end anastomosis would be performed in PV/SMV involved cases.

Postoperative adjuvant chemotherapy included multiagent gemcitabine-based, single-agent gemcitabine based and folfirinox regimens. Patients were followed up in outpatient department or by telephone contact with the patients or their families every 3 months. Post recurrence chemotherapy represented chemotherapy after recurrence was diagnosed, regardless of adjuvant chemotherapy, also with multi-agent gemcitabine-based, single-agent gemcitabine based and folfirinox regimens.

\section{Definitions and data collection}

Preoperative variables were retrieved from the hospital's electronic records system, including age, sex, body mass index (BMI), previous abdominal surgery history, preoperative biliary drainage, total bilirubin, CA19-9 and American Society of Anesthesiologists (ASA) physical status [24], biliary drainage included preoperative biliary stent placement, nasobilliary drainage and percutaneous transhepatic cholangial drainage.

We also obtained data on tumor size defined by the longest diameter of the primary tumor, number of retrieved lymph node, positive lymph nodes with cancer cell metastasis, lymph node ratio which calculated as the number of positive lymph nodes divided by the number of retrieved lymph nodes, TNM stage, R0 resection, lymphovascular invasion and perineural invasion. $\mathrm{R} 0$ resection was defined as absence of malignant cells within $1 \mathrm{~mm}$ from the resection margin using the Royal College of Pathologists definition [25]. Lymphovascular invasion and perineural invasion were based on pathologic report using paraffin sections.

Intraoperative and postoperative variables were also recorded. For RPD cases, docking time was included in the calculation of operative time, estimated blood loss was evaluated based on the vacuum amount, gauze weight and intraoperative fluid infusion volume. Postoperative pancreatic fistula, postpancreatectomy hemorrhage, and delayed gastric emptying represented complications defined by International Study Group on Pancreatic Surgery [1-3]. Biliary fistula was diagnosed by positive drainage of bile acid. Anastomosis fistula was diagnosed by contrastography. Surgical site infections were defined by the Center for Disease Control and Prevention (CDC) definition [26-28], diagnosed by positive pathogen culture in 2 weeks from surgery. Other complications were classified as Clavien-Dindo grade $\geq 3$ other than the complications listed above [29]. 
Follow-up data through September 2019 was retrieved, DFS was calculated from the date of surgery to the date of recurrence or last follow-up if recurrence did not occur. Recurrence was diagnosed by CT or MRI imaging examination. OS was defined as the time from surgery to either death or last follow-up. Patients with death attributed to perioperative morbidity within 90 days or other non-disease-specific reasons in the postoperative period were also censored, only diseasespecific recurrence and disease-specific death were defined as end point events.

\section{Matching}

According to previous reports about the important factors associated with the short-term and long-terms outcomes, together with the variables that would affect the outcomes of RPD and OPD, the propensity score was calculated based on the covariates age, sex, BMI, abdominal surgery history, ASA physical status, CA199, total bilirubin, biliary drainage, tumor size, portal-mesenteric vein resection, year of diagnosis, differentiation, $\mathrm{T}$ and $\mathrm{N}$ stage, lymphovascular invasion and perineural invasion and adjuvant chemotherapy. RPD cases, regardless of conversion to laparotomy $\mathrm{PD}$, were matched to OPD group in a 1:2 ratio and a caliper width of 0.05 standard deviation (SD) was specified.

\section{Statistical analysis}

IBM SPSS Statistics version 24.0 (IBM Corporation) and the statistical packages R (The R Foundation; https://www.r-proje ct.org; version 3.4.3) were used for statistical analysis. Normally distributed continuous variables were presented as mean and SD, and analyzed using Student's $t$-test. Non-normally distributed continuous variables are presented as median and interquartile range (IQR), and analyzed using the Mann-Whitney $U$ test. Categorical variables were presented as frequency or percentage, and analyzed using Chi-square test or Fisher's exact test. Survival analysis of OS and DFS and their corresponding 95\% confidence intervals (CIs) was plotted by the Kaplan-Meier curved and compared with log rank test. Univariate analysis and multivariate COX model analysis were undertaken. When continuous variables were converted to categorical variables, the cutoffs were defined by what previously reported in the literature or by the ROC curve, and variables with $P$ value $<0.1$ in univariate analysis were included into multivariate Cox proportional hazards model for multivariable analysis. $P<0.05$ was considered statistically significant.

\section{Results}

\section{Demographic and baseline characteristics of the study population}

A total of 728 patients underwent RPD or OPD for PDAC during the study period (Fig. 1). 56 were excluded from the final analysis for the following reasons: T4 disease $(n=21)$, distant metastasis $(n=13)$, incomplete data $(n=10)$, and loss to follow up $(n=12)$. The final analysis included 672 subjects (438 male patients, 234 female patients; age at diagnosis: 64 [58-70] years). 115 patients underwent RPD and 557 patients underwent OPD. The demographic and baseline characteristics are presented in Table 1. Compared to the OPD group, the RPD group had significantly lower rate of previous abdominal surgery $(7.0 \%$ vs. $13.5 \%, P=0.032)$, lower preoperative total bilirubin (median [IQR] 29.9 [14.4-94.7] vs. 64.7 [15.8-154.9] $\mu \mathrm{mol} / \mathrm{L}, P<0.001)$, and higher rate of lymphovascular invasion $(58.3 \%$ vs. $47.0 \%, P=0.028)$. The T staging was $\mathrm{T} 1-18.3 \% / \mathrm{T} 2-51.3 \% / \mathrm{T} 3-30.4 \%$ for RPD $v$. T1-19.2\%/T2-48.3\%/T3-32.5\% for OPD $(P=0.840)$. The $\mathrm{N}$ staging was $\mathrm{N} 0-57.4 \% / \mathrm{N} 1-38.3 \% / \mathrm{N} 2-4.3 \%$ for RPD $v s$. N0-53.1\%/N1-36.3\%/N2-10.6\% for OPD $(P=0.115)$.

After propensity score matching at a ratio of 1:2, 105 patients were included in the RPD group and 210 patients in the OPD group.

\section{Intraoperative and perioperative characteristics}

The intraoperative variables and perioperative outcomes are presented in Table 2. Two patients were converted from RPD to OPD because of bleeding and insufficient surgical view, respectively. After matching, the number of retrieved lymph nodes (RPD: 11 [7-16] vs. OPD: 11 [6-17], $P=0.622$ ), number of positive lymph nodes $(0[0-1]$ vs. 0 [0-2], $P=0.975$ ), operative time, estimated blood loss, and length of postoperative hospital stay and the complication rate were all comparable between the two groups. The 30-day mortality rate for RPD was $0 \%$ and $1.0 \%$ for OPD $(P=0.554)$. The 90 -day mortality rate was $1.0 \%$ in both groups.

\section{DFS and OS}

The median follow-up time was 18 (range: 2-74) months.

The Kaplan-Meier DFS and OS curves after matching are shown in Fig. 2.

The median DFS was 14 months (95\% CI 11-22 months) in the RPD group and 12 months (95\% CI 10-14) in the OPD group (HR 0.94; 95\% CI 0.87-1.50; log-rank $P=0.345$ ). 
Fig. 1 The study flowchart

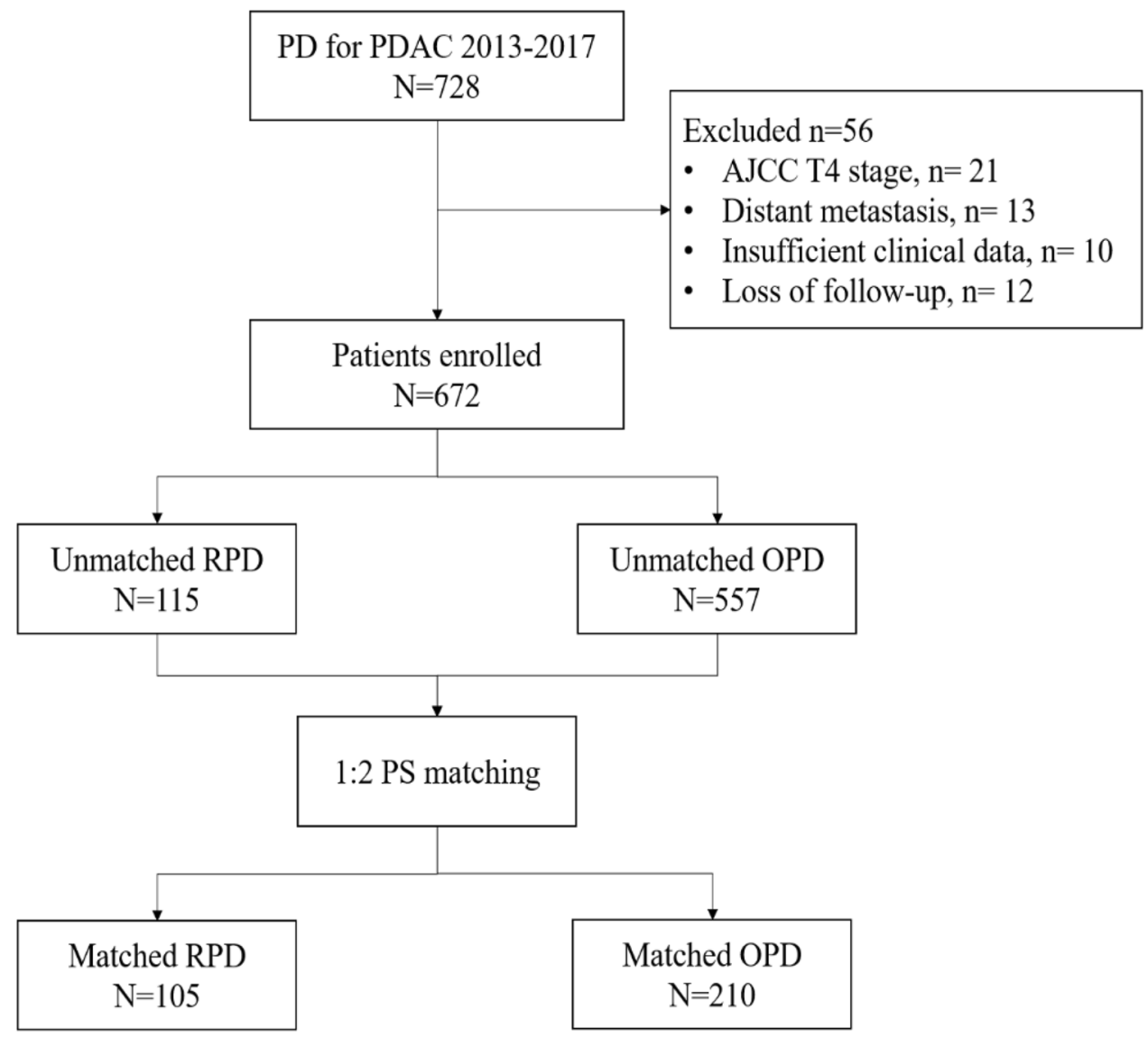

The median OS was 27 months (95\% CI 22-35 months) in the RPD group and 20 months (95\% CI 18-24) in the OPD group (HR 0.77; 95\% CI 0.57-1.04; log-rank $P=0.087$ ).

The 1-year DFS, 3-year DFS, 1-year OS, 3 year OS, the recurrence rate, recurrence patterns and post-recurrence chemotherapy rate are presented in Table 3.

\section{COX regression analysis}

Univariate analysis showed that BMI, CA199 $\geq 300 \mathrm{U} /$ $\mathrm{mL}$, operative time, estimated blood loss, tumor size, T3 stage and R1 resection were associated with DFS $(P<0.10)$ (Table 4). Multivariate analysis with $\mathrm{COX}$ regression model revealed the following independent predictors of DFS: BMI index (HR 0.94; 95\% CI 0.90-0.98; $P=0.008$ ), higher CA199 level ( $>300 \mathrm{U} / \mathrm{mL})(\mathrm{HR} 1.52 ; 95 \% \mathrm{CI}$ $1.14-2.03 ; P=0.005$ ), estimated blood loss (HR 1.00, 95\% CI $1.00-1.01 ; P=0.008)$ and T3 stage (HR $1.82,95 \%$ CI 1.25-2.66; $P=0.002$ ) (Table 4).

Univariate analysis showed that BMI, CA199 $\geq 300 \mathrm{U} /$ $\mathrm{mL}$, estimated blood loss, tumor size, T3 stage, lymphovascular invasion (LVI) and type of surgery were associated with OS $(P<0.10)$ (Table 5). Multivariate analysis with COX regression model revealed the following independent predictors of OS: BMI index (HR 0.94; 95\% CI 0.90-0.99;
$P=0.012)$, high CA199 level (> $300 \mathrm{U} / \mathrm{mL})(\mathrm{HR} 1.56,95 \%$ CI 1.15-2.13; $P=0.005$ ), estimated blood loss (HR 1.00, 95\% CI 1.00-1.00; $P=0.008$ ) and T3 stage (HR 1.71, 95\% CI 1.15-2.54; $P=0.008$ ) (Table 5).

Surgery type (RPD vs. OPD) was not a significant predictor in DFS (HR 0.90; 95\% CI 0.68-1.19; $P=0.456)$ and OS (HR, 0.77; 95\% CI 0.57-1.05; $P=0.094)$.

\section{Discussion}

Since first reported 16 years ago [6], RPD was adopted in pancreatic head, biliary duct and periampullar tumors by some high-volume centers, and its intraoperative and perioperative outcomes was acceptable [4, 12, 30, 31]. Recent studies showed robotic cervical cancer surgery was associated with lower rates of DFS and OS [32, 33], which cause suspicion about the oncological outcomes of other robotic surgery for cancer. In this high volume single-center retrospective study using the propensity score-matching method, we compared the DFS and OS of RPD versus OPD in PDAC patients. Our study demonstrated that patients receiving RPD had comparable DFS and OS versus those undergoing OPD. 
Table 1 Demographic and baseline characteristics of the study population

\begin{tabular}{|c|c|c|c|c|c|c|}
\hline \multirow[t]{2}{*}{ Variables } & \multicolumn{2}{|l|}{ Total cohort } & \multirow[t]{2}{*}{$P$} & \multicolumn{2}{|c|}{ Propensity score matched cohort } & \multirow[t]{2}{*}{$P$} \\
\hline & RPD & OPD & & RPD & OPD & \\
\hline $\mathrm{N}$ & 115 & 557 & & 105 & 210 & \\
\hline Age, years, median (IQR) & $63(57-68)$ & $64(58-70)$ & 0.085 & $64(58-58)$ & $62(58-69)$ & 0.973 \\
\hline Female, $n(\%)$ & $44(38.3 \%)$ & $190(34.1 \%)$ & 0.395 & $39(37.1 \%)$ & $75(35.7 \%)$ & 0.804 \\
\hline BMI, kg/m², mean (SD) & $22.8(2.8)$ & $22.6(3.1)$ & 0.355 & $22.7(2.7)$ & $22.6(3.0)$ & 0.706 \\
\hline Previous abdominal surgery, $n(\%)$ & $8(7.0 \%)$ & $75(13.5 \%)$ & 0.032 & $8(7.6 \%)$ & $15(7.1 \%)$ & 0.878 \\
\hline $\mathrm{TB}, \mu \mathrm{mol} / \mathrm{L}$, median $(\mathrm{IQR})$ & $29.6(14.4-94.7)$ & $64.7(15.8-154.9)$ & $<0.001$ & $36.3(14.9-97.4)$ & $25.4(13.6-118.2)$ & 0.663 \\
\hline Biliary drainage, $n(\%)$ & $36(31.3 \%)$ & $155(27.8 \%)$ & 0.452 & $34(32.4 \%)$ & $51(24.3 \%)$ & 0.127 \\
\hline ASA score $\geq 3, n(\%)$ & $21(18.3 \%)$ & $94(16.9 \%)$ & 0.720 & $19(18.1 \%)$ & $44(21.0 \%)$ & 0.550 \\
\hline CA199, U/mL, median (IQR) & $144.4(40.1-375.4)$ & $153.4(46.0-505.2)$ & 0.264 & $144.4(38.8-434.5)$ & $116.9(26.3-404.4)$ & 0.631 \\
\hline Tumor size, $\mathrm{cm}$, median (IQR) & $3(2.2-3.5)$ & $3.0(2.3-3.8)$ & 0.278 & $3(2.5-3.5)$ & $2.5(2.0-3.6)$ & 0.328 \\
\hline PV/SMV resection, $n(\%)$ & $9(7.8 \%)$ & $53(9.5 \%)$ & 0.569 & $9(8.6 \%)$ & $20(9.5 \%)$ & 0.783 \\
\hline Year of diagnosis, $n(\%)$ & & & $<0.001$ & & & 0.068 \\
\hline 2013 & $3(2.6 \%)$ & $29(5.2 \%)$ & & $3(2.9 \%)$ & $6(2.9 \%)$ & \\
\hline 2014 & $10(8.7 \%)$ & $111(19.9 \%)$ & & $10(9.5 \%)$ & $14(6.7 \%)$ & \\
\hline 2015 & $13(11.3 \%)$ & $170(30.5 \%)$ & & $12(11.4 \%)$ & $43(20.5 \%)$ & \\
\hline 2016 & $36(31.3 \%)$ & $117(21.0 \%)$ & & $36(34.3 \%)$ & $46(21.9 \%)$ & \\
\hline 2017 & $53(46.1 \%)$ & $130(23.3 \%)$ & & $44(41.9 \%)$ & $101(48.1 \%)$ & \\
\hline Poor differentiation, $n(\%)$ & $80(78.3 \%)$ & $418(75.0 \%)$ & 0.465 & $80(76.2 \%)$ & $158(75.2 \%)$ & 0.853 \\
\hline Tumor stage, $n(\%)$ & & & 0.840 & & & 0.388 \\
\hline $\mathrm{T} 1$ & $21(18.3 \%)$ & $107(19.2 \%)$ & & $19(18.1 \%)$ & $47(22.4 \%)$ & \\
\hline $\mathrm{T} 2$ & $59(51.3 \%)$ & $269(48.3 \%)$ & & $53(50.5 \%)$ & $89(42.4 \%)$ & \\
\hline $\mathrm{T} 3$ & $35(30.4 \%)$ & $181(32.5 \%)$ & & $33(31.4 \%)$ & $74(35.2 \%)$ & \\
\hline Lymph node stage, $n(\%)$ & & & 0.115 & & & 0.078 \\
\hline N0 & $66(57.4 \%)$ & $296(53.1 \%)$ & & $57(54.3 \%)$ & $126(60.0 \%)$ & 0.333 \\
\hline N1 & $44(38.3 \%)$ & $202(36.3 \%)$ & & $43(41.0 \%)$ & $63(30.0 \%)$ & \\
\hline $\mathrm{N} 2$ & $5(4.3 \%)$ & $59(10.6 \%)$ & & $5(4.8 \%)$ & $21(10.0 \%)$ & \\
\hline LVI, $n(\%)$ & $67(58.3 \%)$ & $262(47.0 \%)$ & 0.028 & $60(57.1 \%)$ & $112(53.3 \%)$ & 0.522 \\
\hline PNI, $n(\%)$ & $85(73.9 \%)$ & $439(78.8 \%)$ & 0.248 & $79(75.2 \%)$ & $156(74.3 \%)$ & 0.855 \\
\hline Adjuvant chemotherapy, $n(\%)$ & $56(48.7 \%)$ & $283(50.8 \%)$ & 0.680 & $54(51.4 \%)$ & $108(51.4 \%)$ & 1.000 \\
\hline
\end{tabular}

$O P D$ open pancreatoduodenectomy, $R P D$ robotic pancreatoduodenectomy, $B M I$ body mass index, $T B$ total bilirubin, $L V I$ lymphovascular invasion, $P N I$ perineural invasion

Before matching, the RPD group and OPD group differed significantly in previous abdominal surgery history, total bilirubin, year of diagnosis and lymphovascular invasion rate. These may be explained as follows: (1) Surgeons' preference and suggestion of RPD or OPD, to some extent, was determined by patients' total bilirubin index and abdominal surgery history; (2) patients' acceptance of robotic-assisted surgery and proficiency of surgeons in different periods could influence the proportion of patients receiving RPD in different years. However, these differences disappeared after propensity score matching and the other baseline characteristics gained further equivalence to reduce the patientselection bias.

A number of studies about the comparison between RPD and OPD had been published [4, 11, 12, 34], some previous studies showed that main complications such as postoperative pancreatic fistula, biliary fistula, infection and postoperative hemorrhage were not significantly different between minimally invasive pancreatoduodenectomy and OPD [4], some studies even hold a positive attitude towards perioperative short-term outcomes of RPD. In this study, the onset of main complications together with other complications were comparable between the two cohorts. Meanwhile, the estimated blood loss and operative time, which were associated with surgical trauma, were also similar in the two cohorts. The postoperative days, reoperation rate, 30-day and 90-day mortality rate also showed no statistical difference between the two cohorts, prompting us to conclude that the perioperative outcomes of RPD were comparable to those of OPD for PDAC patients. Nevertheless, we cannot neglect that compared with our previous study of RPD [23], when applied in PDAC patients, RPD showed 
Table 2 Intraoperative and perioperative characteristics of the propensity score matched population

\begin{tabular}{|c|c|c|c|c|c|c|}
\hline \multirow[t]{2}{*}{ Variables } & \multicolumn{2}{|l|}{ Total cohort } & \multirow[t]{2}{*}{$P$} & \multicolumn{2}{|c|}{ Propensity score matched cohort } & \multirow[t]{2}{*}{$P$} \\
\hline & RPD & OPD & & RPD & OPD & \\
\hline$N$ & 115 & 557 & & 105 & 210 & \\
\hline Conversion, $n(\%)$ & $2(1.7 \%)$ & - & - & $2(1.8 \%)$ & - & - \\
\hline Retrieved lymph nodes, median (IQR) & $11(6-16)$ & $12(7-18)$ & 0.150 & $11(7-16)$ & $11(6-17)$ & 0.622 \\
\hline Positive lymph nodes, median (IQR) & $0(0-1)$ & $0(0-2)$ & 0.035 & $0(0-1)$ & $0(0-2)$ & 0.975 \\
\hline LNR, median (IQR) & $0(0-0.09)$ & $0(0-0.14)$ & 0.810 & $0(0-0.1)$ & $0(0-0.1)$ & 0.691 \\
\hline$<0.1$ & $89(77.4 \%)$ & $375(67.3 \%)$ & & $79(75.2 \%)$ & $153(72.9 \%)$ & 0.082 \\
\hline$\geq 0.1$ & $26(22.6 \%)$ & $182(32.7 \%)$ & & $26(24.8 \%)$ & $57(27.1 \%)$ & 0.516 \\
\hline R0 resection, $n(\%)$ & $103(89.6 \%)$ & $486(87.3 \%)$ & 0.493 & $93(88.6 \%)$ & $187(89.0 \%)$ & 0.899 \\
\hline Blood loss, mL, median (IQR) & $300(200-500)$ & $300(200-500)$ & 0.746 & $300(200-550)$ & $300(200-500)$ & 0.567 \\
\hline Operative time, min, median (IQR) & $300(245-360)$ & $300(245-335)$ & 0.606 & $300(250-360)$ & $300(240-330)$ & 0.365 \\
\hline POD, d, median (IQR) & $18(14-25)$ & $18(14-26)$ & 0.984 & $17(14-24)$ & $17(14-26)$ & 0.716 \\
\hline Reoperation, $n(\%)$ & $4(3.5 \%)$ & $17(3.1 \%)$ & 0.811 & $4(3.8 \%)$ & $3(1.4 \%)$ & 0.227 \\
\hline 30-day mortality, $n(\%)$ & $0(0 \%)$ & $3(0.5 \%)$ & 0.569 & $0(0 \%)$ & $2(1.0 \%)$ & 0.554 \\
\hline 90-day mortality, $n(\%)$ & $1(0.9 \%)$ & $5(0.9 \%)$ & 0.726 & $1(1.0 \%)$ & $2(1.0 \%)$ & 1.000 \\
\hline POPF & $14(12.2 \%)$ & $70(12.6 \%)$ & 0.908 & $14(13.3 \%)$ & $35(16.7 \%)$ & 0.442 \\
\hline Biochemical leak, $n(\%)$ & $8(7.0 \%)$ & $35(6.3 \%)$ & 0.788 & $8(7.6 \%)$ & $21(10.0 \%)$ & 0.491 \\
\hline CR-POPF, $n(\%)$ & $6(5.2 \%)$ & $35(6.3 \%)$ & 0.664 & $6(5.7 \%)$ & $14(6.7 \%)$ & 0.744 \\
\hline Grade B & $4(3.5 \%)$ & $23(4.1 \%)$ & & $4(3.8 \%)$ & $9(4.3 \%)$ & \\
\hline Grade C & $2(1.7 \%)$ & $12(2.2 \%)$ & & $2(1.9 \%)$ & $5(2.4 \%)$ & \\
\hline Biliary fistula, $n(\%)$ & $6(5.2 \%)$ & $22(3.9 \%)$ & 0.536 & $6(5.7 \%)$ & $11(5.2 \%)$ & 0.860 \\
\hline Anastomotic fistula, $n(\%)$ & $0(0.0 \%)$ & $2(0.4 \%)$ & 0.521 & $0(0.0 \%)$ & $0(0.0 \%)$ & 1 \\
\hline $\mathrm{PPH}, n(\%)$ & $2(1.7 \%)$ & $15(2.7 \%)$ & 0.554 & $2(1.9 \%)$ & $7(3.3 \%)$ & 0.723 \\
\hline Infection, $n(\%)$ & $16(13.9 \%)$ & $84(15.1 \%)$ & 0.749 & $16(15.2 \%)$ & $33(15.7 \%)$ & 0.912 \\
\hline DGE, $n(\%)$ & $4(3.5 \%)$ & $7(1.3 \%)$ & 0.214 & $4(3.8 \%)$ & $2(1.0 \%)$ & 0.098 \\
\hline Others, $n(\%)$ & $8(7.0 \%)$ & $25(4.5 \%)$ & 0.333 & $8(7.6 \%)$ & $11(5.2 \%)$ & 0.403 \\
\hline Total, $n(\%)$ & $31(27.0 \%)$ & $141(25.3 \%)$ & 0.714 & $31(29.5 \%)$ & $58(27.6 \%)$ & 0.723 \\
\hline
\end{tabular}

Others including complications such as pulmonary infection, deep venous thrombosis (DVT), liver abscess, cholangioenteric anastomotic stenosis and chyle leakage Clavien-Dindo Grade $\geq 3$

$O P D$ open pancreatoduodenectomy, $R P D$ robotic-assisted pancreatoduodenectomy, $P O D$ postoperative days, $C R-P O P F$ clinical relevant postoperative pancreatic fistula (ISGPF grade $\mathrm{B}$ and C), $P P H$ post pancreatectomy hemorrhage, $D G E$ delayed gastric emptying

no superiority in perioperative outcomes, which need more samples and specific data collection and analysis on surgical-related prognosis.

The principal object of this study was to compare the oncological outcomes of RPD and OPD for PDAC, and the concerns on the oncological safety of RPD for PDAC can be described as the worries about the ability to have R0 resection and adequate lymphadenectomy in RPD. After matching, variables that might influence DFS and OS such as the number of retrieved and positive lymph nodes, lymph node ratio, lymphovascular invasion and perineural invasion, R0 resection rate, tumor differentiation, $\mathrm{T}$ stage, $\mathrm{N}$ stage, as well as the postoperative adjuvant chemotherapy rate showed no significant difference between the two cohorts, suggesting that the RPD was equally effective in pathological level when compared with OPD. In this study, R0 resection rate in RPD group was $88.6 \%$, not significantly different from that $(89.0 \%)$ in OPD group, besides the pancreatic neck margin, uncinate process and retroperitoneal margins are that surgeons always pay attention to, which means "vascular margin" should not be neglected. There was a remarkable proportion of $\mathrm{PV} / \mathrm{SMV}$ involvement cases in this study. To achieve R0 resection, PV/SMV resection and reconstruction were routinely performed, including wedge resection and repair, end-to-end anastomosis primarily or with gortex grafts. Studies focusing on vascular resection of RPD also showed that vein resection had acceptable perioperative risks and could achieve better survival outcome than that without vein resection $[35,36]$, and PV/SMV involvement was not a contraindication in RPD for PDAC.

Lymphadenectomy was also a crucial point in curative surgery for PDAC patients. The protocol of lymphadenectomy we used in this study depended on the range of Japan Pancreas Society standard lymphadenectomy 
Fig. 2 Kaplan-Meier curves of disease free survival (A log-rank test, $P=0.345$ ) and overall survival (B log-rank test, $P=0.087$ ) of propensity score matched cohorts
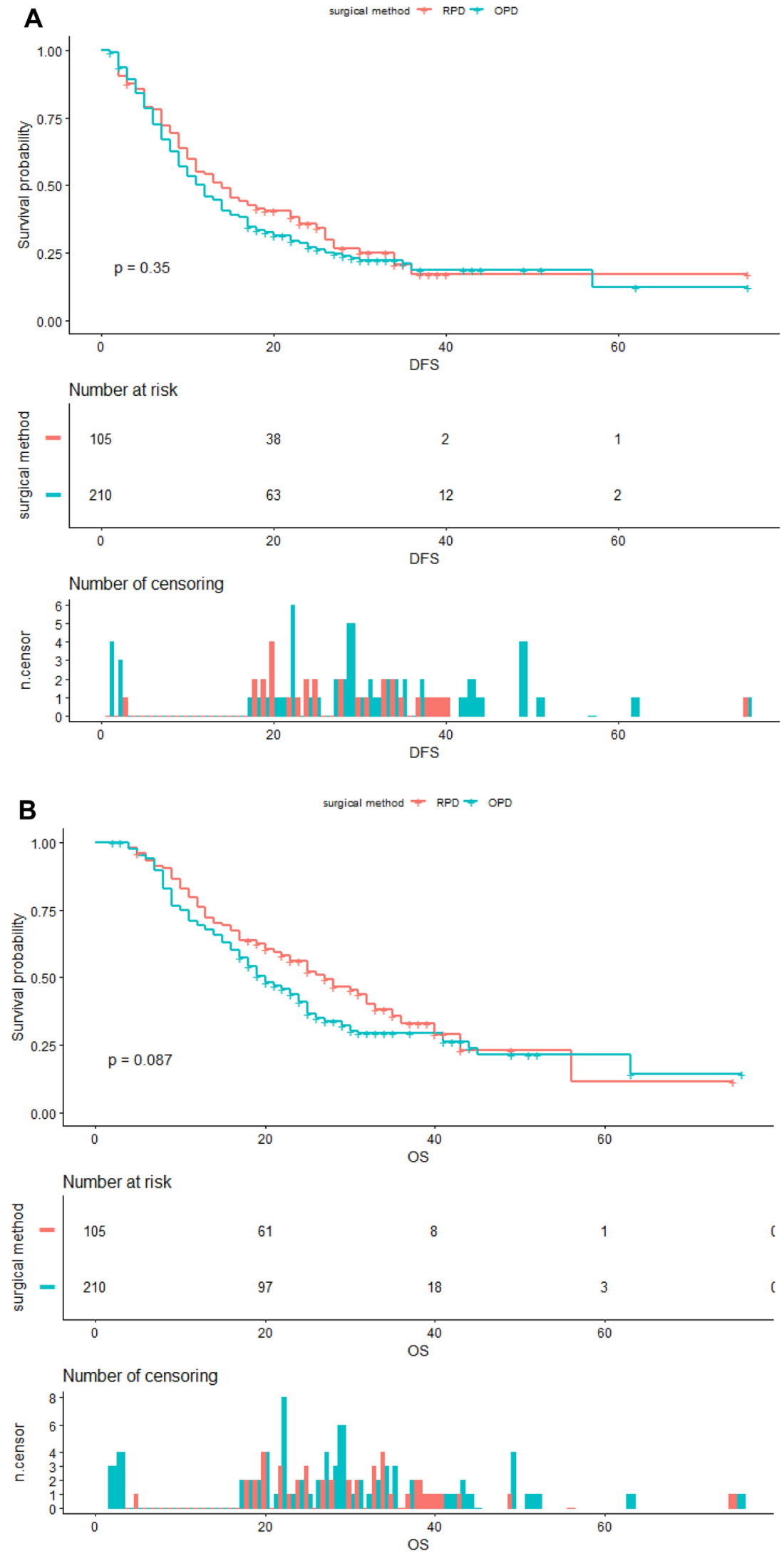
Table 3 Outcomes of the propensity score matched population

\begin{tabular}{llll}
\hline Variables & \multicolumn{2}{l}{ Propensity score matched cohort } & $P$ \\
\cline { 2 - 3 } & RPD $(n=105)$ & OPD $(n=210)$ & \\
\hline DFS & & & 0.345 \\
1-year DFS, (95\% CI) & $53.9 \%(45.1-64.4 \%)$ & $45.9 \%(39.5-53.3 \%)$ & \\
3-year DFS, (95\% CI) & $17.1 \%(9.5-30.8 \%)$ & $18.5 \%(13.1-26.1 \%)$ & \\
Recurrence, $n(\%)$ & $62(59.0 \%)$ & $138(65.7 \%)$ & 0.587 \\
Recurrence pattern, $n(\%)$ & $16(25.8 \%)$ & & \\
Local only & $15(24.2 \%)$ & $41(29.7 \%)$ & 0.572 \\
Liver metastasis only & $16(25.8 \%)$ & $35(25.4 \%)$ & 0.860 \\
Local and liver metastasis & $15(24.2 \%)$ & $50(36.2 \%)$ & 0.147 \\
Other & $43(69.4 \%)$ & $12(8.7 \%)$ & 0.003 \\
Post recurrence chemotherapy & & $75(54.3 \%)$ & 0.046 \\
OS & $76.0 \%(68.2-84.7 \%)$ & $69.5 \%(63.4-76.1 \%)$ & 0.087 \\
1-year OS, (95\% CI ) & $33.0 \%(23.3-46.8 \%)$ & $29.4 \%(23.1-37.3 \%)$ & \\
3-year OS, (95\% CI ) & & & \\
\hline
\end{tabular}

Other, include lung metastasis, bone metastasis, trocar or incision implantation metastasis and malignant ascites

$O P D$ open pancreatoduodenectomy, $R P D$ robotic-assisted pancreatoduodenectomy, $D F S$ disease-free survival, $O S$ overall survival
[37]. In OPD, with tactile sensation, we had better way to explore the positive suspected lymph nodes, and resect these positive suspected lymph nodes subsequently, which eventually induced more advanced $\mathrm{N}$ stage and more positive lymph nodes in the OPD cohort before matching. Compared with OPD, RPD had a different operation view and different approach to perform lymphadenectomy, but with a standard protocol, RPD showed no disadvantages in lymphadenectomy when compared with OPD cohort after matching.

With similar perioperative oncological outcomes and similar adjuvant chemotherapy rate, the Kaplan-Meier survival curve and stratified log-rank test showed that the two cohorts achieved similar DFS $(P=0.345)$. We found three common recurrence patterns in this study, which were local-regional only, liver metastasis only, simultaneous local-regional and liver metastasis, the proportions of these three recurrence patterns were comparable in the two cohorts. Other rare types of recurrence showed difference between the two cohorts, 5(8.1\%) trocar implantation, 7 (11.3\%) lung metastasis, 1 (1.6\%) bone metastasis, $2(3.2 \%)$ malignant ascites were found in RPD cohort, and $4(2.9 \%)$ incision implantation, 7 (5.1\%) lung metastasis, $1(0.7 \%)$ malignant ascites were found in OPD groups. The different post-recurrence chemotherapy rate was an important factor to influence the OS (RPD: 27 [95\% CI 22-35] vs. OPD: 20 [95\% CI 18-24] months (HR 0.77; 95\% CI 0.57-1.04; logrank $P=0.087$ ), patients in RPD cohorts seemed to have better acceptance and tolerance to receive post-recurrence chemotherapy, further studies should be designed to verify the connection between minimally invasive pancreatoduodenectomy and post-recurrence survival.

To investigate survival predictors, we established univariate and multivariate Cox regression analyses model to find out variables associated with DFS and OS. Unadjusted factors significantly associated with DFS and OS (those with $P$ value $<0.1$, were analyzed by multivariable Cox regression model, and BMI, high CA 19-9 level, estimated blood loss and T3 stage proportion were the independent predictors of both DFS and OS, while LVI was an independent risk factor of OS. No significant difference was observed with COX regression model in DFS and OS between RPD and OPD cohorts. These survival analysis results helped us to draw a conclusion that in curative surgery for treatment-naïve PDAC patients, RPD had a non-inferior effect on DFS and OS when compared with OPD.

The study has several limitations. Although the propensity score matching method increased the reliability and credibility, the inevitable selection bias of retrospective study still existed. In addition, due to missing data and loss to follow-up, some cases were eliminated, which led to the reduction in sample capacity, and the limitation of followup period does not allow us to get the complete follow-up data such as the 5-year survival rate. Thirdly, according to our recent study, an important flexion point of RPD learning curve was case No. 250, however considered that excluding the cases in learning curve may cause insufficiency number of cases and follow-up time, we collected cases after the first flexion point of learning curve according to a previous study [20]. Also, neoadjuvant chemotherapy for resectable PDAC was applied in some high-volume center for better survival 
Table 4 Univariate and multivariate cox regression analysis of disease-free survival

\begin{tabular}{|c|c|c|c|c|}
\hline \multirow[t]{2}{*}{ Clinical variations } & \multicolumn{2}{|l|}{ Univariate } & \multicolumn{2}{|l|}{ Multivariate } \\
\hline & HR $(95 \%$ CI) & $P$ value & HR $(95 \%$ CI) & $P$ value \\
\hline Age & $1.00(0.99-1.02)$ & 0.678 & & \\
\hline \multicolumn{5}{|l|}{ Sex } \\
\hline Male & Ref & & & \\
\hline Female & $0.99(0.76-1.30)$ & 0.969 & & \\
\hline BMI & $0.95(0.91-0.99)$ & 0.033 & $0.94(0.90-0.98)$ & 0.008 \\
\hline \multicolumn{5}{|l|}{ CA199 (U/mL) } \\
\hline$<150$ & Ref & & & \\
\hline$\geq 150,<300$ & $1.06(0.72-1.55)$ & 0.774 & $1.06(0.72-1.56)$ & 0.759 \\
\hline$\geq 300$ & $1.49(1.12-1.99)$ & 0.006 & $1.52(1.14-2.03)$ & 0.005 \\
\hline Operative time & $1.00(1.00-1.00)$ & 0.044 & $1.0008(0.999-1.003)$ & 0.463 \\
\hline Estimated blood loss & $1.00(1.00-1.00)$ & 0.005 & $1.00(1.00-1.01)$ & 0.008 \\
\hline Tumor size & $1.19(1.06-1.33)$ & 0.003 & $1.06(0.91-1.23)$ & 0.472 \\
\hline \multicolumn{5}{|c|}{$\mathrm{PV} / \mathrm{SMV}$ reconstruction } \\
\hline No & Ref & & & \\
\hline Yes & $1.25(0.81-1.93)$ & 0.312 & & \\
\hline \multicolumn{5}{|l|}{ T stage } \\
\hline $\mathrm{T} 1$ & Ref & & & \\
\hline $\mathrm{T} 2$ & $1.3455(0.94-1.93)$ & 0.106 & $1.26(0.87-1.81)$ & 0.221 \\
\hline $\mathrm{T} 3$ & $1.93(1.34-2.80)$ & $<0.001$ & $1.82(1.25-2.66)$ & 0.002 \\
\hline \multicolumn{5}{|l|}{$\mathrm{N}$ stage } \\
\hline No & Ref & & & \\
\hline N1 & $0.98(0.74-1.30)$ & 0.902 & & \\
\hline $\mathrm{N} 2$ & $1.10(0.69-1.76)$ & 0.688 & & \\
\hline \multicolumn{5}{|l|}{ Nodal status } \\
\hline $\mathrm{N}-$ & Ref & & & \\
\hline $\mathrm{N}+$ & $1.00(0.77-1.30)$ & 0.971 & & \\
\hline \multicolumn{5}{|l|}{ Examined lymph node } \\
\hline$\leq 8$ & Ref & & & \\
\hline$>8$ & $1.13(0.87-1.48)$ & 0.356 & & \\
\hline \multicolumn{5}{|l|}{ LNR } \\
\hline$<0.1$ & Ref & & & \\
\hline$\geq 0.1$ & $1.05(0.78-1.40)$ & 0.754 & & \\
\hline \multicolumn{5}{|l|}{ Differentiation } \\
\hline Well-intermediate & Ref & & & \\
\hline Poor & $1.13(0.84-1.53)$ & 0.425 & & \\
\hline \multicolumn{5}{|l|}{ Margin } \\
\hline R0 & Ref & & & \\
\hline $\mathrm{R} 1$ & $1.40(0.94-2.08)$ & 0.097 & $1.27(0.85-1.90)$ & 0.246 \\
\hline \multicolumn{5}{|l|}{ LVI } \\
\hline Negative & Ref & & & \\
\hline Positive & $1.19(0.92-1.54)$ & 0.190 & & \\
\hline \multicolumn{5}{|l|}{ PNI } \\
\hline Negative & Ref & & & \\
\hline Positive & $0.96(0.72-1.29)$ & 0.789 & & \\
\hline \multicolumn{5}{|c|}{ Adjuvant chemotherapy } \\
\hline No & Ref & & & \\
\hline Yes & $1.08(0.84-1.40)$ & 0.538 & & \\
\hline \multicolumn{5}{|l|}{ Surgery } \\
\hline OPD & Ref & & & \\
\hline RPD & $0.88(0.67-1.16)$ & 0.359 & $0.90(0.68-1.19)$ & 0.456 \\
\hline
\end{tabular}

Univariable associations $(P<0.10)$ that were selected for multivariable analysis and significant factors $(P<0.05)$ on multivariable analysis are shown in bold 
Table 5 Univariate and multivariate cox regression analysis of overall survival

\begin{tabular}{|c|c|c|c|c|}
\hline \multirow[t]{2}{*}{ Clinical variations } & \multicolumn{2}{|l|}{ Univariate } & \multicolumn{2}{|l|}{ Multivariate } \\
\hline & HR $(95 \%$ CI) & $P$ value & $\mathrm{HR}(95 \% \mathrm{CI})$ & $P$ value \\
\hline Age & $1.00(0.99-1.02)$ & 0.824 & & \\
\hline \multicolumn{5}{|l|}{ Sex } \\
\hline Male & Ref & & & \\
\hline Female & $0.94(0.70-1.26)$ & 0.680 & & \\
\hline BMI & $0.95(0.91-1.00)$ & 0.057 & $0.94(0.89-0.99)$ & 0.012 \\
\hline \multicolumn{5}{|l|}{ CA199 (U/mL) } \\
\hline$<150$ & Ref & & & \\
\hline$\geq 150,<300$ & $1.03(0.67-1.56)$ & 0.907 & $1.06(0.70-1.61)$ & 0.785 \\
\hline$\geq 300$ & $1.48(1.09-2.01)$ & 0.013 & $1.56(1.15-2.13)$ & 0.005 \\
\hline Operative time & $1.00(1.00-1.00)$ & 0.114 & & \\
\hline Estimated blood loss & $1.00(1.00-1.00)$ & 0.006 & $1.00(1.00-1.00)$ & 0.008 \\
\hline Tumor size & $1.17(1.03-1.32)$ & 0.015 & $1.08(0.92-1.25)$ & 0.355 \\
\hline \multicolumn{5}{|c|}{$\mathrm{PV} / \mathrm{SMV}$ reconstruction } \\
\hline No & Ref & & & \\
\hline Yes & $1.36(0.84-2.19)$ & 0.208 & & \\
\hline \multicolumn{5}{|l|}{ T stage } \\
\hline $\mathrm{T} 1$ & Ref & & & \\
\hline $\mathrm{T} 2$ & $1.14(0.77-1.67)$ & 0.510 & $1.14(0.77-1.68)$ & 0.514 \\
\hline $\mathrm{T} 3$ & $1.79(1.21-2.64)$ & 0.003 & $1.71(1.15-2.54)$ & 0.008 \\
\hline \multicolumn{5}{|l|}{$\mathrm{N}$ stage } \\
\hline N0 & Ref & & & \\
\hline N1 & $1.09(0.81-1.48)$ & 0.559 & & \\
\hline $\mathrm{N} 2$ & $0.98(0.58-1.66)$ & 0.951 & & \\
\hline \multicolumn{5}{|l|}{ Nodal status } \\
\hline $\mathrm{N}-$ & Ref & & & \\
\hline $\mathrm{N}+$ & $1.07(0.81-1.42)$ & 0.634 & & \\
\hline \multicolumn{5}{|l|}{ Examined lymph node } \\
\hline$\leq 8$ & Ref & & & \\
\hline$>8$ & $1.21(0.90-1.63)$ & 0.201 & & \\
\hline \multicolumn{5}{|l|}{ LNR } \\
\hline$<0.1$ & Ref & & & \\
\hline$\geq 0.1$ & $1.07(0.78-1.46)$ & 0.687 & & \\
\hline \multicolumn{5}{|l|}{ Differentiation } \\
\hline Well-intermediate & Ref & & & \\
\hline Poor & $0.96(0.69-1.32)$ & 0.786 & & \\
\hline \multicolumn{5}{|l|}{ Margin } \\
\hline R0 & Ref & & & \\
\hline R1 & $1.24(0.80-1.91)$ & 0.341 & & \\
\hline \multicolumn{5}{|l|}{ LVI } \\
\hline Negative & Ref & & & \\
\hline Positive & $1.35(1.01-1.79)$ & 0.040 & $1.45(1.09-1.95)$ & 0.012 \\
\hline \multicolumn{5}{|l|}{ PNI } \\
\hline Negative & Ref & & & \\
\hline Positive & $1.01(0.73-1.39)$ & 0.953 & & \\
\hline \multicolumn{5}{|c|}{ Adjuvant chemotherapy } \\
\hline No & Ref & & & \\
\hline Yes & $1.05(0.79-1.38)$ & 0.743 & & \\
\hline \multicolumn{5}{|l|}{ Surgery } \\
\hline OPD & Ref & & & \\
\hline RPD & $0.77(0.57-1.04)$ & 0.094 & $0.77(0.57-1.05)$ & 0.094 \\
\hline
\end{tabular}

Univariable associations $(P<0.10)$ that were selected for multivariable analysis and significant factors $(P<0.05)$ on multivariable analysis are shown in bold 
outcomes, but was not applied in our center before 2018, so oncological outcomes of RPD vs. OPD for PDAC patients after neoadjuvant chemotherapy still need further analysis. At last, as for sample quantity, the data source was a high volume single center, though with quality control, still not comparable to multicenter studies.

\section{Conclusions}

RPD was comparable to OPD in surgical safety and feasibility, perioperative prognosis, oncological outcomes, and, most importantly, survival outcomes. This study provides important evidence supporting the utilization of RPD in PDAC patients. Based on our work, further prospective randomized controlled studies should be planned to verify the oncological and survival outcomes of RPD in PDAC patients.

\section{Compliance with ethical standards}

Disclosure Yuanchi Weng, Yu Jiang, Ningzhen Fu, Jiabin Jin, Yusheng Shi, Zhen Huo, Xiaxing Deng, Chenghong Peng and Baiyong Shen have no conflicts of interest or financial ties to disclose.

Open Access This article is licensed under a Creative Commons Attribution 4.0 International License, which permits use, sharing, adaptation, distribution and reproduction in any medium or format, as long as you give appropriate credit to the original author(s) and the source, provide a link to the Creative Commons licence, and indicate if changes were made. The images or other third party material in this article are included in the article's Creative Commons licence, unless indicated otherwise in a credit line to the material. If material is not included in the article's Creative Commons licence and your intended use is not permitted by statutory regulation or exceeds the permitted use, you will need to obtain permission directly from the copyright holder. To view a copy of this licence, visit http://creativecommons.org/licenses/by/4.0/.

\section{References}

1. Wente MN, Veit JA, Bassi C et al (2007) Postpancreatectomy hemorrhage (PPH): an International Study Group of Pancreatic Surgery (ISGPS) definition. Surgery 142:20-25

2. Wente MN, Bassi C, Dervenis C et al (2007) Delayed gastric emptying (DGE) after pancreatic surgery: a suggested definition by the International Study Group of Pancreatic Surgery (ISGPS). Surgery 142:761-768

3. Bassi C, Marchegiani G, Dervenis C et al (2017) The 2016 update of the International Study Group (ISGPS) definition and grading of postoperative pancreatic fistula: 11 years after. Surgery 161:584-591

4. McMillan MT, Zureikat AH, Hogg ME et al (2017) A propensity score-matched analysis of robotic vs open pancreatoduodenectomy on incidence of pancreatic fistula. JAMA Surg 152:327-335

5. Cuschieri A (1994) Laparoscopic surgery of the pancreas. J R Coll Surg Edinb 39:178-184
6. Giulianotti PC, Coratti A, Angelini M et al (2003) Robotics in general surgery: personal experience in a large community hospital. Arch Surg 138:777-784

7. Wang SE, Shyr BU, Chen SC et al (2018) Comparison between robotic and open pancreaticoduodenectomy with modified Blumgart pancreaticojejunostomy: a propensity score-matched study. Surgery 164:1162-1167

8. Correa-Gallego C, Dinkelspiel HE, Sulimanoff I et al (2014) Minimally-invasive vs open pancreaticoduodenectomy: systematic review and meta-analysis. J Am Coll Surg 218:129-139

9. Kim HS, Han Y, Kang JS et al (2018) Comparison of surgical outcomes between open and robot-assisted minimally invasive pancreaticoduodenectomy. J Hepatobiliary Pancreat Sci 25:142-149

10. Liu R, Zhang T, Zhao ZM et al (2017) The surgical outcomes of robot-assisted laparoscopic pancreaticoduodenectomy versus laparoscopic pancreaticoduodenectomy for periampullary neoplasms: a comparative study of a single center. Surg Endosc. 31:2380-2386

11. Klompmaker S, van Hilst J, Wellner UF et al (2018) Outcomes after minimally-invasive versus open pancreatoduodenectomy: a pan-European propensity score matched study. Ann Surg. $271: 356$

12. Zureikat AH, Postlewait LM, Liu Y et al (2016) A multi-institutional comparison of perioperative outcomes of robotic and open pancreaticoduodenectomy. Ann Surg 264:640-649

13. Pedziwiatr M, Malczak P, Pisarska M et al (2017) Minimally invasive versus open pancreatoduodenectomy-systematic review and meta-analysis. Langenbecks Arch Surg 402:841-851

14. Nassour I, Choti MA, Porembka MR et al (2018) Robotic-assisted versus laparoscopic pancreaticoduodenectomy: oncological outcomes. Surg Endosc 32:2907-2913

15. Guerra F, Checcacci P, Vegni A et al (2019) Surgical and oncological outcomes of our first 59 cases of robotic pancreaticoduodenectomy. J Visc Surg 156:185-190

16. Coratti A, Di Marino M, Coratti F et al (2016) Initial experience with robotic pancreatic surgery: technical feasibility and oncological implications. Surg Laparosc Endosc Percutan Tech 26:31-37

17. Bencini L, Annecchiarico M, Farsi M et al (2015) Minimally invasive surgical approach to pancreatic malignancies. World $\mathbf{J}$ Gastrointest Oncol 7:411-421

18. Austin PC (2014) The use of propensity score methods with survival or time-to-event outcomes: reporting measures of effect similar to those used in randomized experiments. Stat Med 33:1242-1258

19. von Elm E, Altman DG, Egger M et al (2007) The Strengthening the Reporting of Observational Studies in Epidemiology (STROBE) statement: guidelines for reporting observational studies. Lancet 370:1453-1457

20. Boone BA, Zenati M, Hogg ME et al (2015) Assessment of quality outcomes for robotic pancreaticoduodenectomy: identification of the learning curve. JAMA Surg 150:416-422

21. Tempero MA (2019) NCCN guidelines updates: pancreatic cancer. J Natl Compr Canc Netw 17:603-605

22. Amin MB, Greene FL, Edge SB et al (2017) The Eighth Edition JCC Cancer Staging Manual: Continuing to build a bridge from a population-based to a more "personalized" approach to cancer staging. CA Cancer J Clin 67:93-99

23. Chen S, Chen JZ, Zhan Q et al (2015) Robot-assisted laparoscopic versus open pancreaticoduodenectomy: a prospective, matched, mid-term follow-up study. Surg Endosc 29:3698-3711

24. Kuza CM, Hatzakis G, Nahmias JT (2017) The assignment of American Society of Anesthesiologists physical status classification for adult polytrauma patients: results from a survey and future considerations. Anesth Analg 125:1960-1966

25. Campbell F, Cairns A, Duthie F, et al (2010) Dataset for the histopathological reporting of carcinomas of the pancreas, ampulla of 
Vater and common bile duct from the Royal College of Pathologists. https://www.rcpath.org/resourceLibrary/g091-pancreasda taset-mar17.html.

26. Sawyer RG, Claridge JA, Nathens AB et al (2015) Trial of shortcourse antimicrobial therapy for intraabdominal infection. $\mathrm{N}$ Engl J Med. 372:1996-2005

27. Mazuski JE, Tessier JM, May AK et al (2017) The Surgical Infection Society Revised guidelines on the management of intraabdominal infection. Surg Infect (Larchmt) 18:1-76

28. Weber DG, Bendinelli C, Balogh ZJ (2014) Damage control surgery for abdominal emergencies. Br J Surg 101:e109-118

29. Clavien PA, Barkun J, de Oliveira ML et al (2009) The ClavienDindo classification of surgical complications: five-year experience. Ann Surg 250:187-196

30. Boggi U, Signori S, De Lio N et al (2013) Feasibility of robotic pancreaticoduodenectomy. Br J Surg 100:917-925

31. Bao PQ, Mazirka PO, Watkins KT (2014) Retrospective comparison of robot-assisted minimally invasive versus open pancreaticoduodenectomy for periampullary neoplasms. J Gastrointest Surg 18:682-689

32. Ramirez PT, Frumovitz M, Pareja R et al (2018) Minimally invasive versus abdominal radical hysterectomy for cervical cancer. N Engl J Med 379:1895-1904
33. Melamed A, Margul DJ, Chen L et al (2018) Survival after minimally invasive radical hysterectomy for early-stage cervical cancer. N Engl J Med 379:1905-1914

34. Torphy RJ, Friedman C, Halpern A et al (2018) Comparing shortterm and oncologic outcomes of minimally invasive versus open pancreaticoduodenectomy across low and high volume centers. Ann Surg 270:1147

35. Kauffmann EF, Napoli N, Menonna F et al (2016) Robotic pancreatoduodenectomy with vascular resection. Langenbecks Arch Surg 401:1111-1122

36. Shyr BU, Chen SC, Shyr YM et al (2019) Surgical, survival, and oncological outcomes after vascular resection in robotic and open pancreaticoduodenectomy. Surg Endosc. 34:377

37. Yamaguchi K, Okusaka T, Shimizu K et al (2017) Clinical practice guidelines for pancreatic cancer 2016 from the Japan Pancreas Society: a synopsis. Pancreas 46:595-604

Publisher's Note Springer Nature remains neutral with regard to jurisdictional claims in published maps and institutional affiliations. 